\title{
Neurocardiology: Cardiovascular Changes and Specific Brain Region Infarcts
}

\author{
Rongjun Zou, ${ }^{1}$ Wanting Shi, ${ }^{2}$ Jun Tao, ${ }^{1}$ Hongmu Li, ${ }^{1}$ Xifeng Lin, \\ Songran Yang, ${ }^{3,4,5}$ and Ping Hua ${ }^{1}$ \\ ${ }^{1}$ Department of Cardiovascular Surgery, Sun Yat-sen Memorial Hospital, Sun Yat-sen University, Guangzhou 510120, China \\ ${ }^{2}$ Department of Gastroenterology, The Fifth Affiliated Hospital, Sun Yat-sen University, Zhuhai 519000, China \\ ${ }^{3}$ The Biobank of Sun Yat-sen Memorial Hospital, Sun Yat-sen University, Guangzhou 510120, China \\ ${ }^{4}$ Department of Experimental Psychology, University of Oxford, Oxford OX1 3UD, UK \\ ${ }^{5}$ Guangdong Province Key Laboratory of Brain Function and Disease, Zhongshan School of Medicine, \\ Sun Yat-sen University, Guangzhou 510080, China
}

Correspondence should be addressed to Songran Yang; yangsongran@126.com and Ping Hua; huaping88@sina.com

Received 17 November 2016; Accepted 15 May 2017; Published 3 July 2017

Academic Editor: Vida Demarin

Copyright (C) 2017 Rongjun Zou et al. This is an open access article distributed under the Creative Commons Attribution License, which permits unrestricted use, distribution, and reproduction in any medium, provided the original work is properly cited.

There are complex and dynamic reflex control networks between the heart and the brain, including cardiac and intrathoracic ganglia, spinal cord, brainstem, and central nucleus. Recent literature based on animal model and clinical trials indicates a close link between cardiac function and nervous systems. It is noteworthy that the autonomic nervous-based therapeutics has shown great potential in the management of atrial fibrillation, ventricular arrhythmia, and myocardial remodeling. However, the potential mechanisms of postoperative brain injury and cardiovascular changes, particularly heart rate variability and the presence of arrhythmias, are not understood. In this chapter, we will describe mechanisms of brain damage undergoing cardiac surgery and focus on the interaction between cardiovascular changes and damage to specific brain regions.

\section{Introduction}

Effective cerebral protection remains the principle concern during cardiac surgery. In patients undergoing cardiac surgery, postoperative brain injury can contribute to increased morbidity and mortality and has negative effects on the quality of life and costs. With advances in medical and technological technologies, cardiac surgery can be performed on more complicated cases with several comorbidities. However, the risk of brain injury after surgery is also increased in these patients [1]. For example, heart disease patients show a high incidence of neurological symptoms (40-60\%) including depression and/or depressive symptoms, epilepsy, and forgetfulness [2].

In fact, the primary cause of neurological injury in these patients is thought to relate to formation of an embolus due to gaseous, organic, or inorganic particles generated during the cardiac surgery which lodge in cerebral arteries $[3,4]$. Due to the resulting acute cerebral ischemia, damage to the nucleus tractus solitarius (NTS) can negatively impact on the nucleus ambiguus, dorsal motor nucleus of the vagus, hypoglossal, parabrachial, and locus coeruleus, the hippocampus, entorhinal cortex, prefrontal cortex, amygdala, insula, and many key nuclei in the brainstem. Furthermore, the resulting neuronal and synaptic dysfunction in the NTS may affect its interconnected pathways, impacting on almost the entire central nervous system (CNS) [5]. Other types of structural brain injury, including scattered cerebral infarcts and specific regions of gray matter volume loss, can also occur following cardiac surgery and may contribute to the physiological and behavioral deficits in cardiovascular autonomic responses. For example, cortical autonomic dysfunction may indirectly influence cardiac function via altering centrally mediated autonomic output to the heart in patients with chronic epilepsy [6]. 


\section{Mechanisms of Brain Injury with On-Pump Cardiac Surgery}

The major causes involved in neurologic injury during cardiac surgery are microemboli, hypoperfusion, and inflammatory reaction, which can occur in the $\mathrm{CPB}$ time or any time of perioperative period for a variety of reasons. It has long been known that the brain is highly sensitive to hypoxia and, correspondingly, is threatened by thromboembolic ischemia. With regard to microemboli, the major types causing brain injury are air, fat, and vascular debris, which are distributed in blood flow and cause neuronal injury by blocking cerebral vessels and then leading a hypoperfusion during onpump cardiac surgery [7]. Of note, cerebral microemboli cause larger lesions at higher temperatures, which may be associated with an acute inflammatory reaction.

In this scene, long CPB time initiates a cascade of inflammatory reactions due to the contact of patient blood with external surfaces of extracorporeal circulation machine or surgical stimulation. Additionally, circulating particles directly activate systemic inflammation via contacting with platelets, white blood cells, and endothelial cells. As a result, activated various proinflammatory cytokines, including TNF-alpha, IL-1, IL-6, IL-8, or prostaglandin, are released from platelets, leucocytes, and endothelial cell [8]. Furthermore, another interesting point to discuss is Toll-like receptors (TLRs) [9]. It is widely accepted that TLRs are an important mediator of autophagy and the neuroinflammatory cascade, which contribute to the loss of blood-brain-barrier and behavioral impairment [10, 11]. Endogenous ligands released from ischemic neurons can activate TLR signaling pathways, while release of systemic proinflammatory mediators can activate microglia, resulting in further release of inflammatory cytokines and secondary inflammatory injury [12]. By contrast, the preconditioning phenomena induced by a minor cerebral ischemic event or by TLR ligands can reduce the degree of TLR-mediated inflammation and brain injury following secondary, more severe cerebral ischemia [13].

Additionally, during hypoperfusion, hypoxia leads to anaerobic glycolysis and activation of proapoptotic pathways. There is also a concurrent accumulation of lactate, intracellular calcium overload, and release of reactive oxygen species (ROS), excitatory amino acids (EAA), and inflammatory cytokines in myocytes [14]. ROS are oxygen-derived compounds that serve as a marker for oxidative/nitrosative stress response in relation to brain damage after ischemia. Furthermore, accumulation of ROS could break cellular homeostasis and interfere with cytochrome oxidase, leading to destruction of mitochondria structure and impairment of mitochondrial respiration $[7,8]$. On the other hand, ROS can be generated by arachidonic acid, nitric oxide, catecholamines, glutamate, and activation of N-methyl-daspartate receptors. These compounds lead to an influx of sodium and chloride into neurons, causing secondary intracellular secondary damage, and an influx of water into the cell, resulting in intracellular edema and neuronal death $[1,6,7]$.

In the phase of reperfusion of cerebral lesions, the main energy substrates are dramatically altered; metabolites accumulation causes mitochondrial changes, such as altering the inner membrane potential that disturbs the distribution of ions, leading to intracellular calcium overload, and causing the collapse of mitochondrial membrane structures which opens the mitochondrial permeability transition pore (mPTP) [8]. On one hand, rapid transformation of mitochondria generates an enormous amount of highly reactive and destructive ROS that affect electron transference in the respiratory chain, which dissipates and uncouples the electrochemical gradient of the inner mitochondrial membrane. On the other hand, due to the failure of ATP-dependent ion pumps and intracellular calcium overload, mitochondrial apoptosis-inducing factors (AIF) and cytochrome $\mathrm{C}$ are released during open mPTP. Both molecules are involved in programmed mitochondrial damage and activate the caspase-cascade reactions, which in turn increase intracellular osmolarity and volume and activate apoptin and aggravate the damage of membranaceous structures and organelles $[1,7]$. In summary, it can be stated that these changes can be accompanied by a series of pathways and regulatory molecular mechanisms, such as mitochondrial apoptotic pathways, autophagy, and necrosis.

In fact, in the on-pump cardiac surgery, different pharmacological and nonpharmacological methods to better preserve the brain are still in investigation and many strategies are available to reduce cerebral damage, including adequate anticoagulation, deep hypothermic temperature, or in-depth or screen filters in arterial inflow and venous outflow $[7,8]$.

\section{Predictors of Brain Injury with On-Pump Cardiac Surgery}

In order to identify better indicators of brain injury severity after heart surgery, Özatik et al. compared complement proteins, interleukins, white blood cells, and $S 100 \beta$ protein before the initiation of $\mathrm{CPB}$, immediately prior to aortic cross-clamping, following unclamping, and at postoperative 1st and 24th hours in 40-patient prospective cohort study. The authors found that high serum level of $S 100 \beta$ protein is associated with increased levels of serum inflammatory mediators and systemic inflammatory response during $\mathrm{CPB}$ [15]. Additionally, Demir et al. analysed thirty patients' serum neuron-specific enolase (NSE), interleukin-6 (IL-6), and IL-10 before CPB and 4 and 24 hours after the end of extracorporeal circulation and found that serum cytokine and NSE levels were significantly associated with neuronal damage [16].

However, whether serum neuromarkers (NSE, S100 $\beta$ ) have a predictive effect on the adverse neurological outcome has not been concluded yet. Sanchez-de-Toledo et al. measured cerebral regional oxygen saturation ( $\mathrm{rSO} 2)$ and serum biomarkers such as NSE, S100 $\beta$, glial fibrillary acidic protein (GFAP), and brain-derived neurotrophic factor (BDNF) in 39 neurologically normal patients undergoing cardiac surgery with $\mathrm{CPB}$. They prospectively observed adverse neurological outcomes via pediatric cerebral performance category (PCPC) score at 12 months after surgery and found that cerebral rSO2 was significantly associated with neurological 
impairment, while none of the serum neuromarkers (NSE, S100 $\beta$, GFAP, and BDNF) in this cohort appear to be helpful [17]. Similarly, the explorative analysis from a randomized clinical trial, SafeBoosC II, showed that none of the S100 $\beta$, brain-fatty-acid-binding-protein, and neuroketal were associated with the burden of either cerebral hypoxia or hyperoxia [18]. As described above, there is a debate on the effect of serum neuromarkers (NSE, S100 $\beta$ ) in adverse neurological outcome with on-pump cardiac surgery.

\section{Interaction between Cardiovascular Changes and Specific Brain Regions}

In 1985, Natelson [19] described a new interdisciplinary area termed "neurocardiology," which examines the interaction between the cardiovascular and autonomic nervous systems in pathological states. Modern neuroimaging data, including positron emission tomography and functional magnetic resonance imaging, show a complex set of neural interactions termed the neurocardiac axis, consisting of the insular cortex, the anterior cingulate cortex, the prefrontal cortex, and the amygdala, an area connected to other regions involved in autonomic control $[20,21]$. Baroreflex sensitivity, dynamic electrocardiogram (ECG) changes, and heart rate variability (HRV) are also important parameters in understanding the influence of the autonomic system on both heart and brain activity [22].

4.1. Cardiovascular Changes and the Insular Cortex. As the insular cortex is located in the region of the middle cerebral arteries, it is more susceptible to injury following cerebrovascular disease. Indeed, $\sim 33 \%$ of patients with hemispheric cerebral ischemia exhibited an insular infarct [23]. Damage to the insular cortex damage is associated with arrhythmia, disruption in diurnal blood pressure variation, myocardial injury, and breathing disorders during sleep [24], as well as elevated plasma levels of brain natriuretic peptide, catecholamines, and neuropeptide Y (NPY) [25, 26].

The insular cortex is a core region for interoceptive processing, including cardiac perception, emotion, and awareness, and for self-consciousness [27, 28]. The insular cortex also plays a crucial role in the central autonomic network and is typically associated with autonomic sympathetic activation [29]. In imaging studies examining cortical gray matter volumes, a smaller size of the bilateral insula predicted the magnitude of autonomic change following infarction [30]. The alterations in interoceptive or exteroceptive environmental cues observed in psychiatric disorders can also have serious cardiovascular consequences, including inhomogeneous ventricular repolarizing current, sinus node pacemaker current, and bundle-branch block. It also results in a variety of cardiac changes, including electrophysiological, structural, and contractile dysfunction and sudden cardiac death [21].

Cardiovascular autonomic dysfunction is mainly related to increased sympathetic activity via the right hemisphere of the insular cortex [31]. Alterations in autonomic nervous system function have been linked to ventricular and atrial arrhythmias. For example, both sympathetic-controlled
HRV and parasympathetic-controlled HRV were decreased in patients with right hemisphere stroke involving reduced bilateral insula volumes $[30,32]$. Further, these patients were more susceptible to cardiac complications such as arrhythmias and sudden death due to autonomic imbalance $[33,34]$.

The ECG changes observed following cerebral ischemia may be mediated through abnormal autonomic discharges caused by increased levels of brain natriuretic peptide, catecholamines, and NPY [21, 35, 36]. NPY released by sympathetic neurons can reduce vagal neurotransmission and directly influence ventricular myocyte excitability. NPY can also inhibit acetylcholine release during vagal nerve stimulation and bind to NPY receptors located on cholinergic ganglia and ventricular myocytes to inhibit $\beta$-receptor activity $[37,38]$. NPY is widely distributed in both the central and peripheral nervous systems and is functionally related to regulation of blood pressure and circadian rhythms. Further, as a potent growth factor, NPY can cause cell proliferation in multiple systems, especially in the cardiovascular system [36], which is associated with cardiac hypertrophy via increased protein synthesis [39] and innervation-dependent changes in catecholamine-dependent chronotropic responsiveness [40]. Finally, there is increasing evidence that neurons can release NPY after myocardial infarction, which is involved in the progress of bilateral interventions targeting the sympathetic chain for arrhythmia modulation [24, 25, 41].

The stellate ganglia play an important role in ventricular and atrial arrhythmia following insular cortex infarction. Structural changes in the stellate ganglion and cardiac nervous system occur as a result of chronic increases in sympathetic input and can cause arrhythmogenesis [42]. T wave changes are a key marker of ventricular tachyarrhythmia, myocardial infarction, and sudden cardiac death and are related to dispersion of repolarization during stellate ganglia activation $[43,44]$. Circulating norepinephrine did not affect the ventricular dispersion of repolarization [42]. Further, atrial fibrillation, atrioventricular block, ectopic beats, sinus bradycardia, inverted $\mathrm{T}$ wave, and sudden cardiac death were more common in patients with a right insular lesion than in those with a left insular lesion. However, patients with bilateral acute insular lesions typically showed atrial fibrillation, third-degree block, ST depression, and inverted $\mathrm{T}$ waves [45]. Thus, it remains unclear whether the ECG changes associated with insular infarcts are related to the size of the insular lesion, as only partial insular infarcts or total insular infarcts have been taken into account [35] (Figure 1).

4.2. Cardiovascular Changes and the Brainstem. The brainstem (i.e., the external lateral parabrachial nucleus within the pons and rostral ventrolateral medulla) contributes to CNS processing of excitatory cardiovascular reflexes during cardiac sympathetic stimulation [46, 47]. The brainstem is an important regulator of cardiovascular responses to the interactive or exteroceptive environment, as well as vagal and sympathetic nerve activity [48]. Patients complicated with cardiac autonomic dysfunction due to brainstem lesions can exhibit ventricular arrhythmias, inverted $\mathrm{T}$ wave, myocardial 

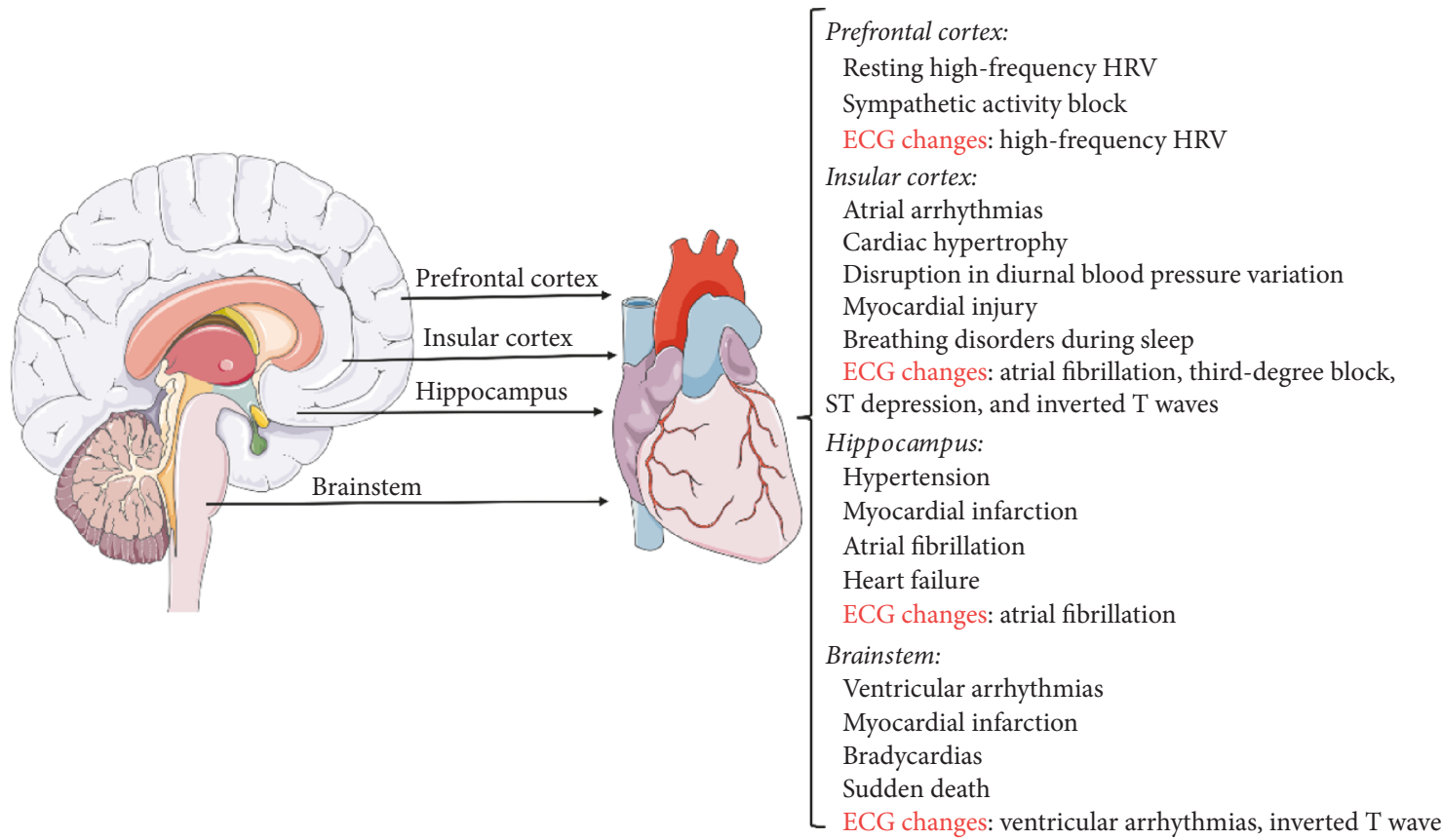

FIGURE 1: This figure shows that central and peripheral mechanism of the heart and brain interaction includes the clinical symptoms and ECG changes, but the effect of stellate ganglion or vagus and sympathetic nerves is not shown in this figure for an illustration. Of note, insular cortex, prefrontal cortex, hippocampus, and brainstem play an important role in interplay between the nervous system and the cardiovascular system.

infarction, bradycardias, and even sudden death $[20,24,25$, 48] (Figure 1).

4.3. Cardiovascular Changes and the Prefrontal Cortex. Resting high-frequency HRV (HF-HRV) is considered to reflect vagal blocking of sympathetic activity and can be used to assess the interaction of cardiovascular changes with the nervous system following cerebral injury after surgery. Interestingly, individual differences in resting HF-HRV can vary with resting state neural activity in the prefrontal cortex [49]. The prefrontal cortex region is of particular interest, as connectivity related to HF-HRV is shared by the default nervous network. However, increasing evidence suggests that HF-HRV is not directly related to the global resting state activity of intrinsic brain networks but rather to more localized connectivity. Thus, impaired autonomic cardiac control may occur following injury to brain regions such as the prefrontal cortex. For example, using a combination of electroencephalographic dynamics and instantaneous HR estimates to study emotional processing and cardiovascular autonomic responses in brain areas such as the prefrontal cortex and amygdala infract, Valenza et al. [50] found significant arousal-dependent changes between positive and negative stimuli, especially at intermediate arousing levels, throughout the prefrontal cortex (Figure 1).

4.4. Cardiovascular Changes and the Hippocampus. There is evidence that widespread hippocampal infarcts are associated with clinical symptoms of cognitive impairment or epilepsy. In a meta-analysis, Ishibashi et al. reported that the hippocampus was particularly vulnerable to ischemic insults under ultralow flow cardiopulmonary bypass [51]. Drabek et al. reported significant changes in the cortex, thalamus, hippocampus, and amygdala/piriform complex over time following early cerebral hyperperfusion and delayed cerebral hypoperfusion in rats [52]. Further, multiple mediators that accumulate during cerebral ischemia, as well as systemic factors such as acidosis, EAA, inflammatory factors, and ROS, have been implicated in both the no-flow and resuscitation periods $[51,53,54]$.

A number of cardiovascular risk factors, including hypertension, myocardial infarction, atrial fibrillation, and heart failure, are also associated with large hemispheric brain infarcts [55]. Nevertheless, hippocampal infarcts play a prominent role, with postmortem evidence of large hemispheric brain infarcts being associated with heart failure and altered cardiovascular index [56, 57]. This is likely because large hemispheric infarcts, cardiovascular disease, and hippocampal infarcts have common risk factors [55], while the hippocampus and some other brain regions are particularly susceptible to cerebral ischemia and neuronal degeneration, resulting in the clinical syndrome of cognitive impairment, myocardial infarction, and heart failure [58] (Figure 1).

\section{Summary}

Improving our understanding of the mechanisms regulating postoperative brain injury and cardiovascular changes will aid in the development of appropriate therapeutic measures 
for cardiac surgery patients. Careful management of the operation period and favorable cardiopulmonary bypass conditions, as well as control of temperature, flow rate, $\mathrm{pH}$, and hematocrit levels, can reduce the risk of cerebral infarction and thus avoid autonomic nerve damage and improve the prognosis of patients with cardiac surgery [59]. Similarly, furthering our understanding of the brain-heart axis and how brain lesions result in dynamic changes in ECG and HRV is important for facilitating the treatment of clinical symptoms in patients with brain injury after cardiac surgery.

\section{Conflicts of Interest}

The authors have no conflicts of interest to disclose.

\section{Acknowledgments}

This work was supported by the Natural Science Foundation Project in Guangdong Province, China (Grant no. 2016A030313295) and the Major Project Development and Emerging, Interdisciplinary Funding Projects of Sun Yat-sen University (Grant no. 15ykjc17b).

\section{References}

[1] S. Scolletta, F. S. Taccone, and K. Donadello, "Brain injury after surgery," Minerva Anestesiol, vol. 81, no. 6, pp. 662-677, 2015.

[2] W. Jiang, M. Kuchibhatla, G. L. Clary, M. S. Cuffe, E. J. Christopher, J. D. Alexander et al., "Relationship between depressive symptoms and long-term mortality in patients with heart failure," American Heart Journal, vol. 154, no. 1, pp. 102108, 2007.

[3] I. B. Hovens, B. L. van Leeuwen, M. A. Mariani, A. D. Kraneveld, and R. G. Schoemaker, "Postoperative cognitive dysfunction and neuroinflammation; Cardiac surgery and abdominal surgery are not the same," Brain, Behavior, and Immunity, vol. 54, pp. 178-193, 2016.

[4] P. A. Barber, S. Hach, L. J. Tippett, L. Ross, A. F. Merry, and P. Milsom, "Cerebral ischemic lesions on diffusion-weighted imaging are associated with neurocognitive decline after cardiac surgery," Stroke, vol. 39, no. 5, pp. 1427-1433, 2008.

[5] I. Ay, R. Nasser, B. Simon, and H. Ay, "Transcutaneous cervical vagus nerve stimulation ameliorates acute ischemic injury in rats," Brain Stimulation, vol. 9, no. 2, pp. 166-173, 2016.

[6] K. Ravindran, K. L. Powell, M. Todaro, and T. J. O’Brien, “The pathophysiology of cardiac dysfunction in epilepsy," Epilepsy Research, vol. 127, pp. 19-29, 2016.

[7] A. Salameh, S. Dhein, I. Dähnert, and N. Klein, "Neuroprotective strategies during cardiac surgery with cardiopulmonary bypass," International Journal of Molecular Sciences, vol. 17, no. 11, p. E1945, 2016.

[8] J. W. Hammon, "Brain protection during cardiac surgery: Circa 2012," Journal of Extra-Corporeal Technology, vol. 45, no. 2, pp. 116-121, 2013.

[9] Z.-X. Tang, G.-X. Chen, M.-Y. Liang et al., "Selective antegrade cerebral perfusion attenuating the TLR4/NF- $\kappa$ B pathway during deep hypothermia circulatory arrest in a pig model," Cardiology (Switzerland), vol. 128, no. 3, pp. 243-250, 2014.
[10] P. Mukherjee, C. W. Winkler, K. G. Taylor et al., "SARM1, not MyD88, mediates TLR7/TLR9-induced apoptosis in neurons," Journal of Immunology, vol. 195, no. 10, pp. 4913-4921, 2015.

[11] F. Gilli, L. Li, and A. R. Pachner, "The immune response in the CNS in Theiler's virus induced demyelinating disease switches from an early adaptive response to a chronic innatelike response," Journal of Neuro Virology, vol. 22, no. 1, pp. 66-79, 2016.

[12] H. Shi, N. Gabarin, E. Hickey, and R. Askalan, “TLR-3 receptor activation protects the very immature brain from ischemic injury," Journal of Neuroinflammation, vol. 10, article no. 104, 2013.

[13] P.-F. Wang, X.-Y. Xiong, J. Chen, Y.-C. Wang, W. Duan, and Q.-W. Yang, "Function and mechanism of toll-like receptors in cerebral ischemic tolerance: From preconditioning to treatment," Journal of Neuroinflammation, vol. 12, no. 1, article no. 80, 2015.

[14] F. P. González-Ibarra, J. Varon, and E. G. López-Meza, “Therapeutic hypothermia: critical review of the molecular mechanisms of action," Frontiers in Neurology, vol. 3, article 4, 2011.

[15] M. A. Özatik, O. Tarcan, A. Kale et al., "Do $\$ 100 \beta$ protein level increases due to inflammation during cardiopulmonary bypass occur without any neurological deficit?" Perfusion, vol. 17, no. 5, pp. 335-338, 2002.

[16] T. Demir, H. Demir, T. Tansel et al., "Influence of methylprednisolone on levels of neuron-specific enolase in cardiac surgery: A corticosteroid derivative to decrease possible neuronal damage," Journal of Cardiac Surgery, vol. 24, no. 4, pp. 397-403, 2009.

[17] J. Sanchez-De-Toledo, C. Chrysostomou, R. Munoz et al., "Cerebral regional oxygen saturation and serum neuromarkers for the prediction of adverse neurologic outcome in pediatric cardiac surgery," Neurocritical Care, vol. 21, no. 1, pp. 133-139, 2014.

[18] A. M. Plomgaard, T. Alderliesten, T. Austin, F. van Bel et al., "Early biomarkers of brain injury and cerebral hypo- and hyperoxia in the SafeBoosC II trial," Plos One, vol. 12, no. 3, p. e0173440, 2017.

[19] B. H. Natelson, "Neurocardiology: an Interdisciplinary Area for the 80s," Archives of Neurology, vol. 42, no. 2, pp. 178-184, 1985.

[20] D. S. Goldstein, "Sympathetic neuroimaging," in Handbook of Clinical Neurology, vol. 117, pp. 365-370, Elsevier, 2013.

[21] K. J. Lizarraga, A. Gorgulho, W. Chen, and A. A. Salles, "Molecular imaging of movement disorders," World Journal of Radiology, vol. 8, no. 3, p. 226, 2016.

[22] K. Shivkumar, O. A. Ajijola, I. Anand, J. A. Armour, P.-S. Chen, M. Esler et al., "Clinical neurocardiology defining the value of neuroscience-based cardiovascular therapeutics," Journal of Physiology, vol. 594, no. 14, pp. 3911-3954, 2016.

[23] M. Pasquini, C. Laurent, M. Kroumova, I. Masse, D. Deplanque, $\mathrm{X}$. Leclerc et al., "Insular infarcts and electrocardiographic changes at admission: Results of the PRognostic of Insular CErebral infarctS Study (PRINCESS)," Journal of Neurology, vol. 253, no. 5, pp. 618-624, 2006.

[24] S. Oppenheimer and D. Cechetto, "The insular cortex and the regulation of cardiac function," Comprehensive Physiology, vol. 6, no. 2, pp. 1081-133, 2016.

[25] M. Nagai, S. Hoshide, and K. Kario, "The insular cortex and cardiovascular system: a new insight into the brain-heart axis," Journal of the American Society of Hypertension, vol. 4, no. 4, pp. 174-182, 2010. 
[26] S. Koppikar, A. Baranchuk, J. C. Guzmán, and C. A. Morillo, "Stroke and ventricular arrhythmias," International Journal of Cardiology, vol. 168, no. 2, pp. 653-659, 2013.

[27] R. Ronchi, J. Bello-Ruiz, M. Lukowska, B. Herbelin, I. Cabrilo, K. Schaller et al., "Right insular damage decreases heartbeat awareness and alters cardio-visual effects on bodily selfconsciousness," Neuropsychologia, vol. 70, pp. 11-20, 2015.

[28] J. L. Ardell, M. C. Andresen, J. A. Armour, G. E. Billman, P.S. Chen, R. D. Foreman et al., "Translational neurocardiology: preclinical models and cardioneural integrative aspects," Journal of Physiology, vol. 594, no. 14, pp. 3877-3909, 2016.

[29] F. Colivicchi, A. Bassi, M. Santini, and C. Caltagirone, "Cardiac autonomic derangement and arrhythmias in right-sided stroke with insular involvement," Stroke, vol. 35, no. 9, pp. 2094-2098, 2004.

[30] E. Makovac, F. Meeten, D. R. Watson, S. N. Garfinkel, H. D. Critchley, and C. Ottaviani, "Neurostructural abnormalities associated with axes of emotion dysregulation in generalized anxiety," NeuroImage: Clinical, vol. 10, pp. 172-181, 2016.

[31] G. Orlandi, S. Fanucchi, G. Strata, L. Pataleo, L. Landucci Pellegrini et al., "Transient autonomic nervous system dysfunction during hyperacute stroke," Acta Neurologica Scandinavica, vol. 102, no. 5, pp. 317-321, 2000.

[32] E. Makovac, F. Meeten, D. R. Watson, A. Herman, S. N. Garfinkel et al., "Alterations in amygdala-prefrontal functional connectivity account for excessive worry and autonomic dysregulation in generalized anxiety disorder," Biological Psychiatry, vol. 80, no. 10, pp. 786-795, 2016.

[33] T. B. Alves, L. T. Totola, A. C. Takakura, E. Colombari, and T. S. Moreira, "GABA mechanisms of the nucleus of the solitary tract regulates the cardiovascular and sympathetic effects of moxonidine," Autonomic Neuroscience: Basic and Clinical, vol. 194, pp. 1-7, 2016.

[34] S. Meyer, M. Strittmatter, C. Fischer, T. Georg, and B. Schmitz, "Lateralization in autononic dysfunction in ischemic stroke involving the insular cortex," NeuroReport, vol. 15, no. 2, pp. 357361, 2004.

[35] D. Michalski, C. Weise, C. Hobohm, L. Küppers-Tiedt, J. Pelz, D. Schneider et al., "Autonomic reactions and peri-interventional alterations in body weight as potential supplementary outcome parameters for thromboembolic stroke in rats," Experimental and Translational Stroke Medicine, vol. 4, no. 1, article no. 7, 2012.

[36] P. M. Masliukov, K. Moiseev, A. I. Emanuilov, T. A. Anikina, A. A. Zverev, A. D. Nozdrachev et al., "Development of neuropeptide Y-mediated heart innervation in rats," Neuropeptides, vol. 55, pp. 47-54, 2016.

[37] N. Herring, "Autonomic control of the heart: Going beyond the classical neurotransmitters," Experimental Physiology, vol. 100, no. 4, pp. 354-358, 2015.

[38] M. D. P. Heredia, C. Delgado, L. Pereira, R. Perrier, S. Richard, G. Vassort et al., "Neuropeptide Y rapidly enhances $\left[\mathrm{Ca}^{2+}\right] \mathrm{i}$ transients and $\mathrm{Ca}^{2+}$ sparks in adult rat ventricular myocytes through Y 1 receptor and PLC activation," Journal of Molecular and Cellular Cardiology, vol. 38, no. 1, pp. 205-212, 2005.

[39] R. Zhang, H. Niu, X. Kang, T. Ban, H. Hong, and J. Ai, "Longterm administration of neuropeptide $\mathrm{y}$ in the subcutaneous infusion results in cardiac dysfunction and hypertrophy in rats," Cellular Physiology and Biochemistry, vol. 37, no. 1, pp. 94-104, 2015.
[40] H.-A. Chang, W.-H. Fang, T.-C. Chang, S.-Y. Huang, and C.C. Chang, "Association of neuropeptide y promoter polymorphism (rs16147) with perceived stress and cardiac vagal outflow in humans," Scientific Reports, vol. 6, Article ID 31683, 2016.

[41] O. A. Ajijola, D. Yagishita, N. K. Reddy et al., "Remodeling of stellate ganglion neurons after spatially targeted myocardial infarction: neuropeptide and morphologic changes," Heart Rhythm, vol. 12, no. 5, pp. 1027-1035, 2015.

[42] B. L. Nguyen, H. Li, M. C. Fishbein, S.-F. Lin, C. Gaudio, P.S. Chen et al., "Acute myocardial infarction induces bilateral stellate ganglia neural remodeling in rabbits," Cardiovascular Pathology, vol. 21, no. 3, pp. 143-148, 2012.

[43] M. Vaseghi, K. Yamakawa, A. Sinha, So. EL, W. Zhou, O. A. Ajijola et al., "Modulation of regional dispersion of repolarization and T-peak to T-end interval by the right and left stellate ganglia," American Journal of Physiology-Heart and Circulatory Physiology, vol. 305, no. 7, pp. H1020-H1030, 2013.

[44] D. Yagishita, R. W. Chui, K. Yamakawa, P. S. Rajendran, O. A. Ajijola, K. Nakamura et al., "Sympathetic nerve stimulation, not circulating norepinephrine, modulates T-peak to T-end interval by increasing global dispersion of repolarization," Circulation: Arrhythmia and Electrophysiology, vol. 8, no. 1, pp. 174-185, 2015.

[45] H. Christensen, G. Boysen, A. F. Christensen, and H. H. Johannesen, "Insular lesions, ECG abnormalities, and in outcome in acute stroke," Journal of Neurology, Neurosurgery and Psychiatry, vol. 76, no. 2, pp. 269-271, 2005.

[46] Z. L. Guo, J. C. Longhurst, S. C. Tjen-A-Looi, and L. W. Fu, "elPBN neurons regulate rVLM activity through elPBN-rVLM projections during activation of cardiac sympathetic afferent nerves," American Journal of Physiology - Regulatory, Integrative and Comparative Physiology, vol. 311, no. 2, pp. R410-R425, 2016.

[47] V. Constantinescu, D. Matei, D. Cuciureanu, C. Corciova, B. Ignat, and C. D. Popescu, "Cortical modulation of cardiac autonomic activity in ischemic stroke patients," Acta Neurologica Belgica, vol. 116, no. 4, pp. 473-480, 2016.

[48] K. J. Cummings, K. G. Commons, K. C. Fan, A. Li, and E. E. Nattie, "Severe spontaneous bradycardia associated with respiratory disruptions in rat pups with fewer brain stem 5HT neurons," American Journal of Physiology - Regulatory Integrative and Comparative Physiology, vol. 296, no. 6, pp. R1783-R1796, 2009.

[49] J. R. Jennings, L. K. Sheu, D. C.-H. Kuan, S. B. Manuck, and P. J. Gianaros, "Resting state connectivity of the medial prefrontal cortex covaries with individual differences in high-frequency heart rate variability," Psychophysiology, vol. 53, no. 4, pp. 444454, 2016.

[50] G. Valenza, A. Greco, C. Gentili, A. Lanata, L. Sebastiani, D. Menicucci et al., "Combining electroencephalographic activity and instantaneous heart rate for assessing brain-heart dynamics during visual emotional elicitation in healthy subjects," Philosophical Transactions of the Royal Society A: Mathematical, Physical and Engineering Sciences, vol. 374, no. 2067, 2016.

[51] N. Ishibashi, Y. Iwata, T. Okamura, D. Zurakowski, H. G. Lidov, and R. A. Jonas, "Differential neuronal vulnerability varies according to specific cardiopulmonary bypass insult in a porcine survival model," The Journal of Thoracic and Cardiovascular Surgery, vol. 140, no. 6, pp. 1408-1415.e3, 2010.

[52] T. Drabek, L. M. Foley, A. Janata, J. Stezoski, T. Kevin Hitchens, M. D. Manole et al., "Global and regional differences in cerebral blood flow after asphyxial versus ventricular fibrillation cardiac arrest in rats using ASL-MRI," Resuscitation, vol. 85, no. 7, pp. 964-971, 2014. 
[53] H. W. Lin, V. L. Gresia, H. M. Stradecki, A. Alekseyenko, C. Dezfulian, J. T. Neumann et al., "Protein kinase C delta modulates endothelial nitric oxide synthase after cardiac arrest," Journal of Cerebral Blood Flow and Metabolism, vol. 34, no. 4, pp. 613-620, 2014.

[54] Y. Xu, S. M. Liachenko, P. Tang, and P. H. Chan, "Faster recovery of cerebral perfusion in SOD1-overexpressed rats after cardiac arrest and resuscitation," Stroke, vol. 40, no. 7, pp. 2512-2518, 2009.

[55] T. Rauramaa, M. Pikkarainen, E. Englund, P. G. Ince, K. Jellinger, A. Paetau et al., "Cardiovascular diseases and hippocampal infarcts," Hippocampus, vol. 21, no. 3, pp. 281-287, 2011.

[56] B. Wandschneider, M. Koepp, C. Scott, C. Micallef, S. Balestrini, S. M. Sisodiya et al., "Structural imaging biomarkers of sudden unexpected death in epilepsy," Brain, vol. 138, no. 10, pp. 29072919, 2015.

[57] S. L. Bealer and J. G. Little, "Seizures following hippocampal kindling induce QT interval prolongation and increased susceptibility to arrhythmias in rats," Epilepsy Research, vol. 105, no. 1-2, pp. 216-219, 2013.

[58] K. A. Jellinger, "The pathology of 'vascular dementia': a critical update," Journal of Alzheimer's Disease, vol. 14, no. 1, pp. 107-123, 2008.

[59] V. Anttila, I. Hagino, D. Zurakowski, Y. Iwata, L. Duebener, H. G. W. Lidov et al., "Specific bypass conditions determine safe minimum flow rate," Annals of Thoracic Surgery, vol. 80, no. 4, pp. 1460-1467, 2005. 


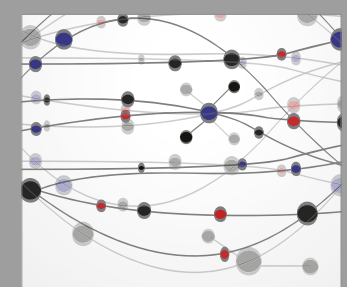

The Scientific World Journal
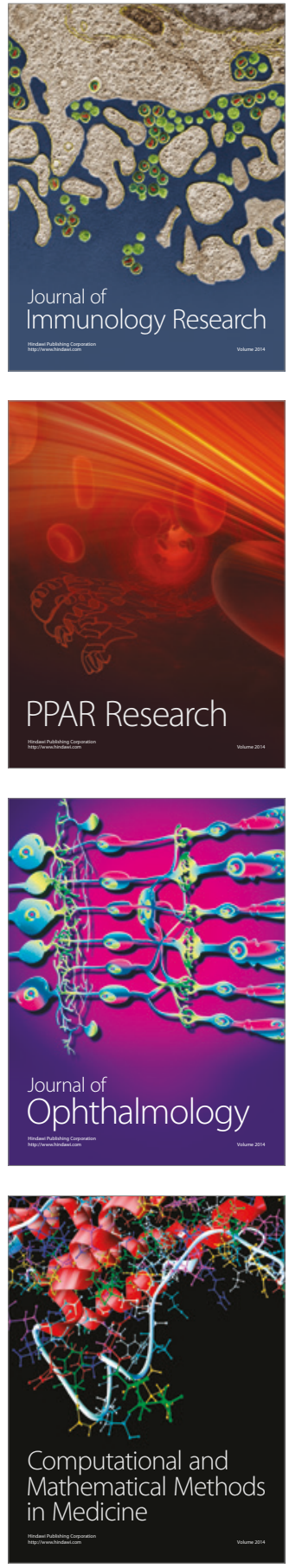

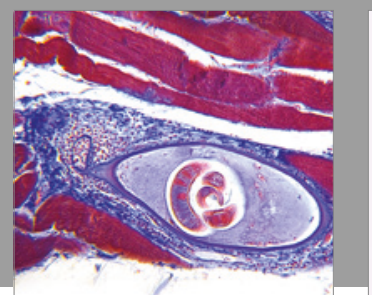

Gastroenterology Research and Practice
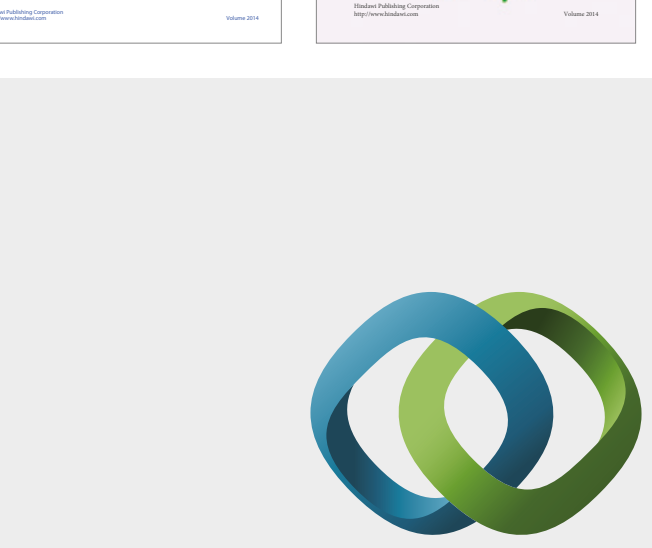

\section{Hindawi}

Submit your manuscripts at

https://www.hindawi.com
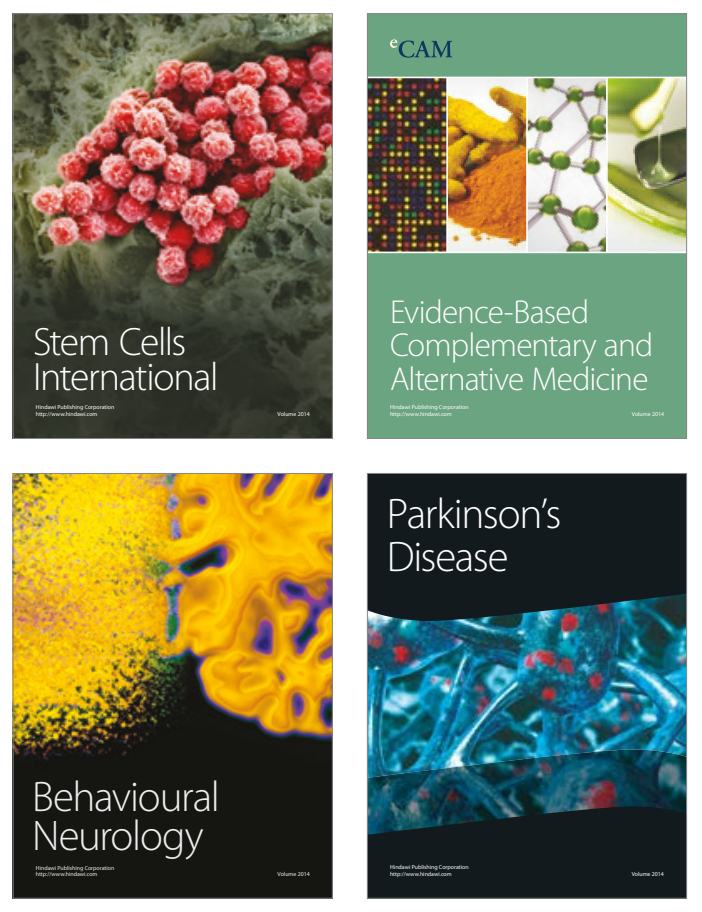
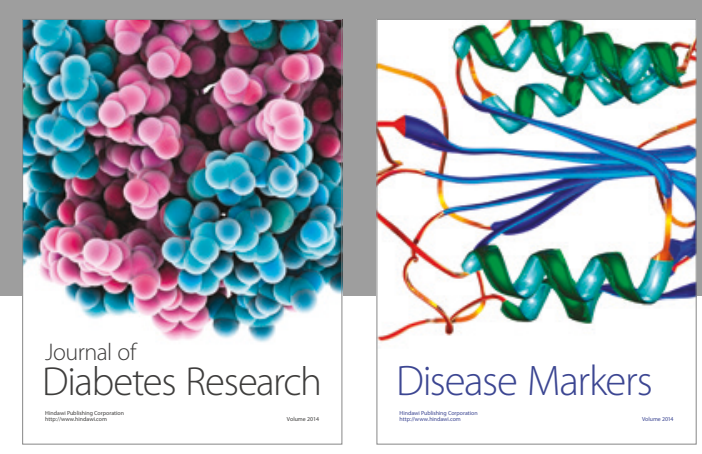

Disease Markers
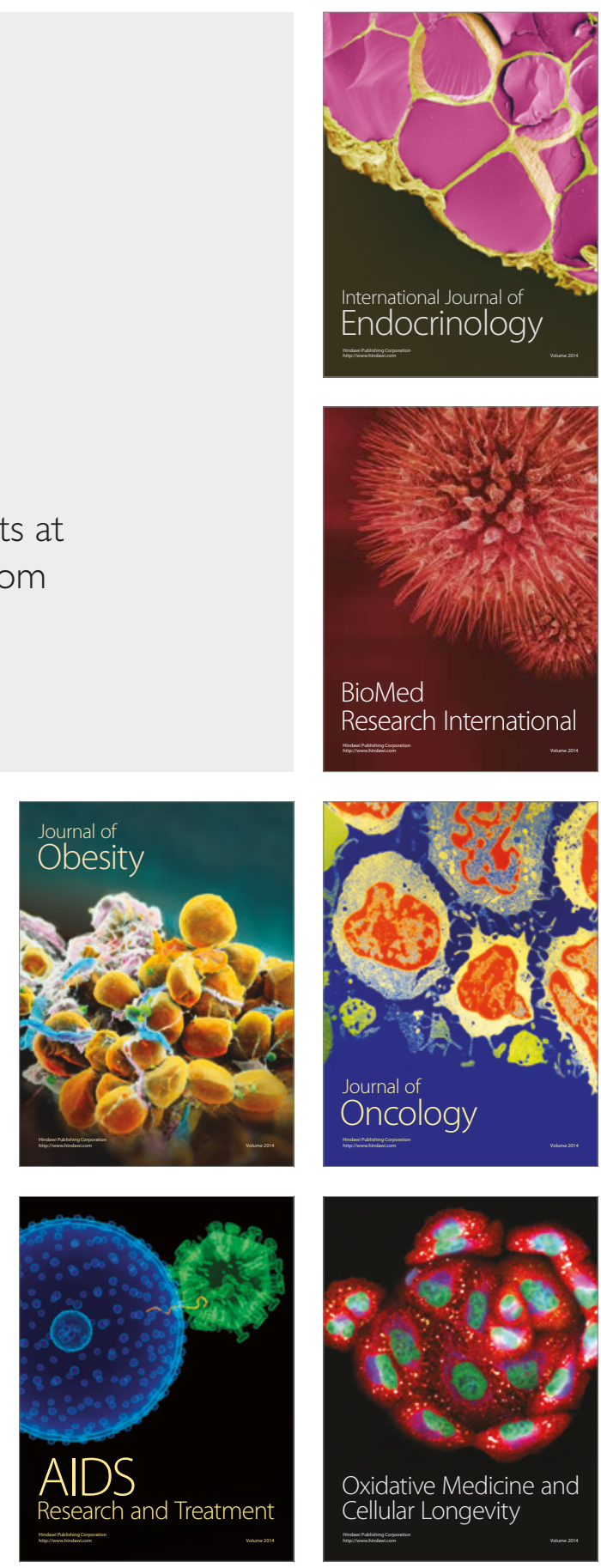